<Oleomaterial Award 2021>

\title{
Engineering Advanced Composite Materials Using Liquid Crystals: From Device Application to Unconventional Cosmetics
}

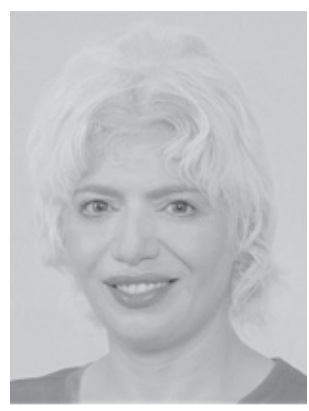

Fatin HAJJAJ

Research and Development Department, Chifure Holdings Corporation

2-2-4 Minamidai, Kawagoe-shi, Saitama 350-1165, Japan

\begin{abstract}
Mixing two individually well-known materials is an established route to engineering composites with tailored properties. Such composite materials, namely those based on liquid crystals, offer the possibility of exploring phase behaviors in condensed soft matter, leading to exotic phenomena and technological innovations. This contribution reviews the design and application of advanced liquid crystal-based composites, which show emergent and intriguing properties that stem from interactions with strong magnetic fields or confinement in condensed polymer systems. This review should be of interest to academics and industries interested in magneto-responsive materials, non-volatile memory, and innovative approaches to protect and beautify the skin.
\end{abstract}

Key words: liquid crystal composites, magnetic field, diamagnetic materials, magnetotropic phase behavior, PDLC film, lyotropic lipid mesophases, second skin barrier, unconventional cosmetics

\section{Introduction}

The design of soft matter that responds with emergent or synergistic behavior to a local signal, as changes in humidity or fields, remains a contemporary challenge, one of the reasons being that its responsiveness is hardly predictable ${ }^{1)}$. A primary motivation for creating such material arises from the great potential in technological innovations because its unusual responsiveness is often associated with totally new material properties that can revolutionize the industry. Typically, composites combining the responsiveness of a liquid crystalline (LC) phase with the robustness of a metal or polymer scaffold constitute the optimal materials for exploring new functionalities in soft matter. Such composites can promote the formation of stochastic or intermediate states, which often exhibit instability and unusual response behaviors under external stimuli ${ }^{2-4)}$.

連絡者：Fatin Hajjaj

E-mail : fatin_hajjaj@chifuregrp.co.jp
Beyond the simple preparation of composites that combine the additive value of the individual components, this review discusses the designing strategy to generate emergent properties and performance in soft condensed materials. In particular, LC-based composites where an intermediate mesophase leads to unusual behavior induced by magnetic fields and how confining a lyotropic mesophase in a condensed polymer system can cause significant changes in the polymer's intrinsic properties.

Soft condensed systems are pliable combinations of liquids and solids, which have rich phase diagrams, exhibit nonlinear responses, and are strongly affected by thermal fluctuations ${ }^{1)}$. These systems are abundant, encompassing biomaterials (e.g., skin tissues), polymers, gels, LCs, and their composites. Various material processing technologies use external forces or fields to silence the thermal randomization in such complex systems and achieve control over the phase transition behavior. Among all the stimuli that can be applied to 
manipulate the material properties, the magnetic field is the most effective as it offers a massively parallel manipulation approach. Magnetic fields enable the manipulation of magnetic and nonmagnetic materials with length scales ranging from millimeter down to the nanometer, and due to their contactless and noninvasive nature, they can safely penetrate most materials, including biological objects. In contrast, conventional stimuli as light and electric fields tend to degrade the materials by inducing redox and electrochemical reactions and are generally considered harmful to biological materials, including the skin. By exploiting the interactions between magnetic fields and diamagnetic materials, leading scientists have discovered exotic phenomena and demonstrated new soft-matter technologies. In the light of these advances, the review begins with a brief description of the interaction between magnetic fields and materials with a diamagnetic response (i.e., a ubiquitous material property that accounts for only a weak response to the magnetic field). Beyond the conventional alignment responses, recent advances have focused on engineering adaptive systems capable of leveraging the weak diamagnetic interactions to achieve significant responses involving unparalleled performance and emergent behavior. One such advance is the capability to control the molecular structure of organic matter with magnetic fields, which is reviewed in detail, focusing on the material design, response mechanism, and application in device technology.

More recently, efforts have shifted toward engineering composites that display amplification of the properties of the individual components or multifunctionality. Even more intriguing is the possibility of combining two different materials so that a new physical property not observed in the individual materials emerges. Polymers can be, in principle, engineered to promote emergent and synergistic properties in soft condensed matter. Given the potential for a functional synergism and emergence afforded by the use of polymers to confine LCs while simultaneously regulating their phase behavior, significant opportunities exist for exploring LCpolymer composites to create high-performance materials. The simple and scalable approach for creating such advanced composites is the polymer-dispersed liquid crystal (PDLC), which is compatible with the material processing technologies commonly used in the cosmet- ic, pharmaceutical, and food industries. In PDLC composites, where isolated LC microdroplets are dispersed in a polymer matrix, novel optical and mechanical properties are realizable by changing the polymer structure or the LC phase ${ }^{5)}$. The large surface-to-volume ratio of PDLC films leads to many film characteristics being dominated by surface, rather than bulk, properties of the $\mathrm{LC}^{5}$. When considering the cosmetic functions of the lyotropic LC materials ${ }^{6}$, ranging from optical effects to moisturizing, PDLC films can provide unconventional cosmetics due to their highly functional surface and the collective effects of millions of LC microdroplets synergized with the polymer properties. In this context, PDLC films functioning as a second skin barrier would be of great interest. The next section of the review addresses functional beauty films based on PDLC, highlighting their formulation, structural and surface features, skin performance, and cosmetic application.

The review concludes by discussing future directions of the presented work and the current challenges and opportunities in using the diamagnetic response of biological soft materials in skin research. Finally, it is necessary to point out that this article focuses on recent advances achieved by the author and does not intend to be a comprehensive review of the literature.

\section{Engineering Advanced Responsive Composites: Exceptional Properties via Magnetic Field Interac- tions}

\section{1 Interaction between magnetic fields and diamagnetic soft composite}

Interactions between fields and matter represent the origin of functional expression in soft condensed materials. Magnetic field interactions play a vital role in manipulating the physical properties of ferromagnetic and paramagnetic soft matter ${ }^{7}$. Such materials possess a spin (i.e., magnetic moment due to unpaired electrons) and therefore show a relatively strong response to magnetic fields compared to diamagnetic materials, which are devoid of spin. Diamagnetism arises from a tiny distortion of the electron orbits within atoms or molecules by applied magnetic fields. This quantum mechanical effect occurs in all materials, and unlike ferro- and paramagnetism, it always makes a weak contribution to the material's response to a magnetic field. 
Consequently, strong magnetic fields $(>1 \mathrm{~T})$ are required to manipulate diamagnetic materials to generate a significant magnetic response. Strong magnetic fields tend to repel diamagnetic materials causing them to align, deform, separate, or levitate to minimize their magnetic energy ${ }^{7-9}$. These magnetic responses often modify the bulk properties of diamagnets, but they cannot affect small molecular aggregates, where changes in the magnetic energy are too small to silence the thermal randomization or overcome other energetic barriers $^{7-9}$. Since most soft materials are diamagnetic, effective design strategies that harness diamagnetic responses to generate a new material property would be of great technological importance.

Soft composite systems, often associated with complex dynamics and response, can be engineered to generate new material properties through diamagnetic interactions. In pursuing these objectives, Liu et al. designed a hydrogel composite reinforced with charged titanate (metal-oxide) nanosheets as a filler ${ }^{10)}$. When subjecting a colloidal aqueous dispersion of the titanate nanosheets to a strong magnetic field $(10 \mathrm{~T})$, the diamagnetic sheets aligned cofacially, giving rise to maximum repulsive interactions among the sheets. The dispersion was then converted into a hydrogel using in situ polymerization, which suppressed thermal relaxation and locked in the anisotropic electrostatic feature of the filler. The obtained magnetically structured hydrogel showed unusual mechanical anisotropy reminiscent of articular cartilage, where directional vibrations are damped to achieve a frictionless motion even under high compression. A combination of polymer and LC can also provide unique functional properties through diamagnetic responses. Rešetič et al. have shown that exceptional thermomechanical response is achievable in PDLC composites by imprinting their shape memory anisotropy using a strong (9 T) orienting magnetic field ${ }^{11)}$. In these composite systems, changes in the magnetic orientation of the diamagnetic LC filler are manifested macroscopically as programmable morphing functionalities. These magnetically structured composites are promising materials for additive manufacturing and 3D printing technologies.

\subsection{Diamagnetic LC composites with unusual phase behavior induced by magnetic field interactions}

As noted, the bulk properties of diamagnets can be modified by magnetic field interactions, mainly through alignment responses that can manifest macroscopically in soft composites as, for example, unusual mechani$\mathrm{cal}^{10)}$, optical ${ }^{10)}$, or thermomechanical ${ }^{11)}$ properties. However, in such magneto-responsive materials, the assembled structures of molecules themselves remain intact, most likely because the energy of a magnetic field is generally too small to bias the mode of molecular assembly under thermodynamic control. Accordingly, the resulting structures at the molecular level are usually independent of the presence or absence of a magnetic field, even when a strong magnetic field generated from a superconducting magnet is applied. Beyond the alignment and deformation responses ${ }^{8)}$, although magnetic fields are known to change the chiral ${ }^{9)}$ and polymorphic (i.e., isomerism) ${ }^{12,13)}$ structures of organic matter during nucleation, crystal growth, and solidification processes, the occurrence of a magneto-induced structural selection was considered impossible (vide supra). If it occurs, it should bring about unique material property and exotic phenomena as it involves changes in the diamagnet molecular structure.

Recently, Hajjaj et al. have demonstrated an unprecedented phase behavior in a diamagnetic molecular assembly, which emerges during thermal processing in strong magnetic fields ${ }^{4}$. Crucial to this discovery were the experimental approach (vide infra) and the design of an LC composite system with a rich phase diagram. Depending on the temperature (thermotropic behavior) and metal content (metallotropic behavior), the LC composite system showed a phase diagram where cubic, columnar orthorhombic, and columnar hexagonal mesophases emerge in the absence of a magnetic field. Figure 1 provides a quick overview of what happened to a diamagnetic LC composite interacting with a strong magnetic field. When a diamagnetic LC composite $\left({ }^{\mathrm{Im}} \mathrm{TP}[\mathrm{La}]_{x}\right.$; Fig. 1a) was thermally processed in the presence of strong magnetic fields ( $>5 \mathrm{~T}$ ), its original phase diagram was dramatically changed (Fig. 1b), where the orthorhombic (Ortho) phase was completely replaced by a cubic (Cub) phase. This unexpected phenomenon was discovered accidentally during alignment experiments on a paramagnetic analog ${ }^{\mathrm{Im}} \mathrm{TP}[\mathrm{Dy}]_{x}$; Fig. 1a), where a particular type of LC assembly selectively develops a thermodynamically disfavored Cub phase when processed under a strong magnetic field by cool- 

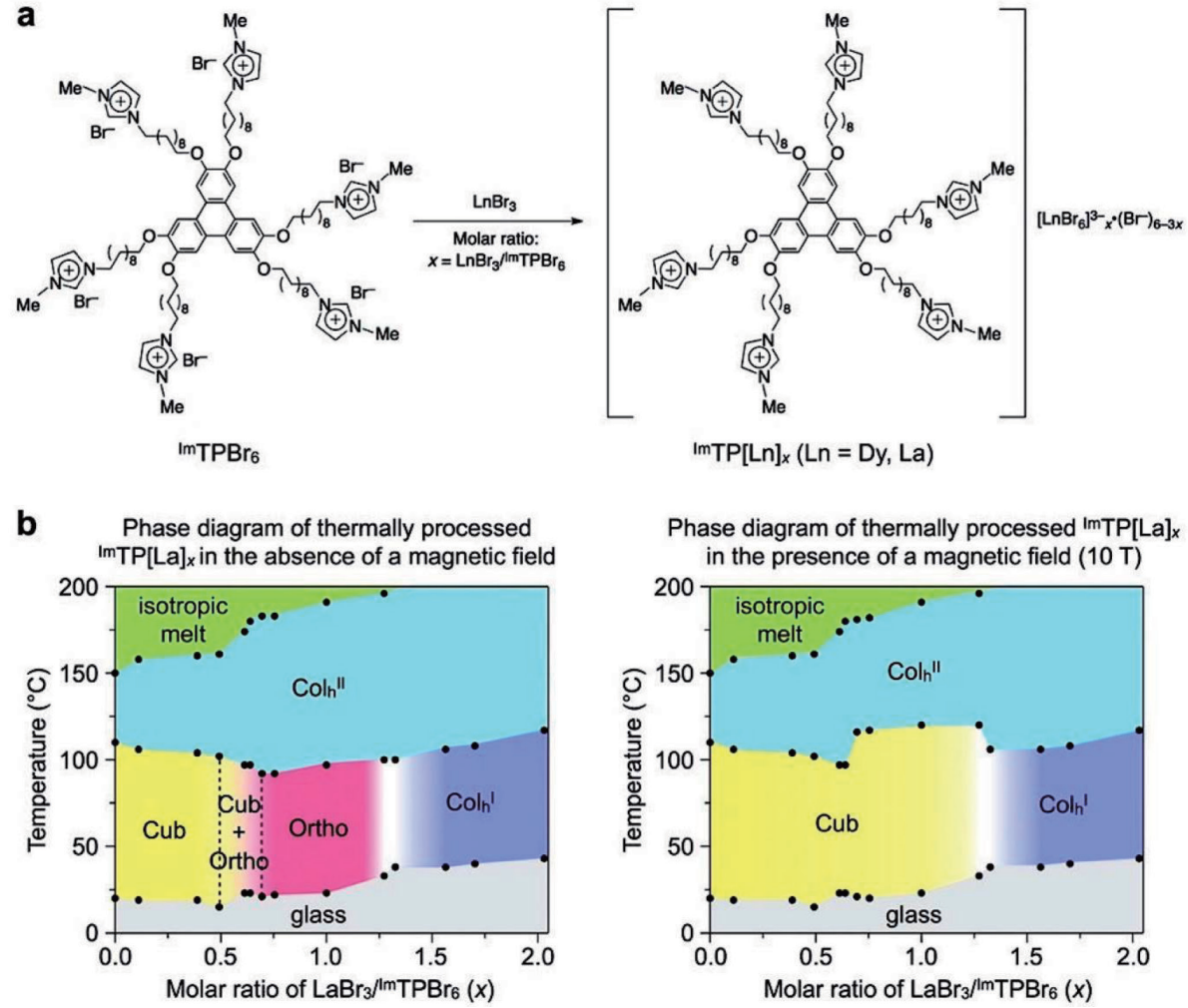

Fig. 1 (a) Preparation of the LC composites $\left({ }^{\mathrm{Im}} \mathrm{TP}[\mathrm{Ln}]_{x}\right.$ ) by mixing ${ }^{\mathrm{Im}} \mathrm{TPBr}_{6}$, a discotic ionic $\mathrm{LC}$ material ${ }^{14)}$, with different molar ratios $(x)$ of a paramagnetic $\left(\mathrm{DyBr}_{3}\right)$ or diamagnetic $\left(\mathrm{LaBr}_{3}\right)$ lanthanide $(\mathrm{Ln})$ salt. (b) Phase diagrams of the diamagnetic LC composite $\left({ }^{\mathrm{Im}} \mathrm{TP}[\mathrm{La}]_{x}\right)$, obtained after thermal processing in the absence or presence of a 10-T magnetic field. Cub: cubic, Ortho: orthorhombic, $\mathrm{Col}_{\mathrm{h}}$ : hexagonal columnar. The boundaries of the white regions could not be clearly determined. Figure reproduced from ref. (4) with permission from Springer Nature.

ing from its hot melt. Surprisingly, even the diamagnetic LC composite $\left({ }^{\mathrm{Im}} \mathrm{TP}[\mathrm{La}]_{x}\right)$ displayed a similar magneto-assisted phase selection, confirming that this phenomenon arises due to diamagnetic responses of the LC assembly.

The phase diagrams of ${ }^{\mathrm{Im}} \mathrm{TP}[\mathrm{La}]_{x}$ (Fig. 1b) were established based on analyses using synchrotron X-ray diffraction (XRD; Fig. 2), differential scanning calorimetry (DSC), and polarized optical microscopy (POM; Fig. 2). Notably, the intermediate Ortho phase (Fig. 2c), which appears in the phase diagram of ${ }^{\mathrm{Im}} \mathrm{TP}[\mathrm{La}]_{x}$ in the absence of a magnetic field (Fig. 1b), is not a kinetically formed metastable phase but rather a thermodynamically stable $\mathrm{LC}^{4)}$. However, interactions with strong fields $(>5 \mathrm{~T}$ ) destabilize such thermodynamically preferred LC, forcing its high-temperature hexagonal phase $\left(\mathrm{Col}_{h}{ }^{\mathrm{II}}\right)$ to selectively develop a Cub LC (Fig. 2e) as the low-temperature phase. Interestingly, the magnetically selected Cub phase (Fig. 2e) is very stable at ambient conditions and does not transform back to the original Ortho phase, even when kept for 3 months at $25^{\circ} \mathrm{C}$ outside the magnet. Here, the initial Ortho phase re-emerges only by thermal processing the magnetically selected Cub phase without a magnetic field (Fig. 2f). This erasable memorized phase selection is a unique feature of this LC composite system. Unlike most adaptive systems that usually undergo thermal relaxation and require a fixation treatment (e.g., polymerization) to trap the transiently induced structures or effects to ambient conditions ${ }^{10,11}$. Most likely, the highly viscous nature of the ${ }^{\mathrm{Im}} \mathrm{TP}[\mathrm{La}]_{x}$ composite, which is responsible for the glass phase formation at low temperatures (Fig. 1b), assisted in locking in the magneto-induced Cub phase (Fig. 2e) emerged during cooling in strong magnetic fields.

\subsection{Discovery of a magnetotropic phase behavior and applications in a nonvolatile memory device}

To reveal the magneto-assisted structural selection mechanism, Hajjaj et al. designed homemade instruments and dedicated experimental setups (Fig. 3a) for directly monitoring the LC phase behavior under strong magnetic fields). In situ POM observations (Fig. 

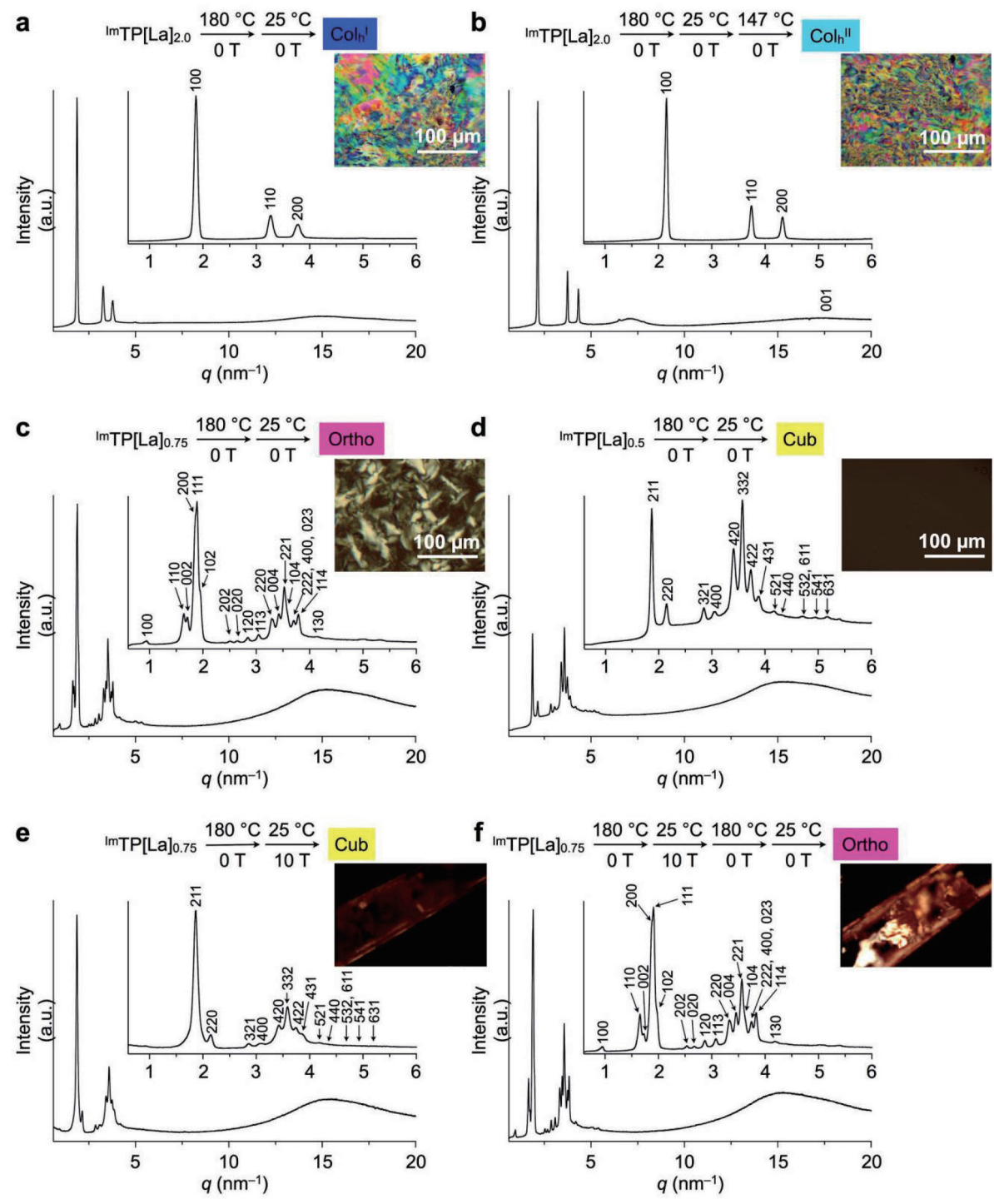

Fig. 2 XRD patterns and magnifications of the small-angle region (scattering vector $q=0.6-6 \mathrm{~nm}^{-1}$ ) of (a) ${ }^{\mathrm{Im}} \mathrm{TP}[\mathrm{La}]_{2.0}$ at $27^{\circ} \mathrm{C}$ on first heating, (b) ${ }^{\mathrm{Im}} \mathrm{TP}[\mathrm{La}]_{2.0}$ at $147^{\circ} \mathrm{C}$ on first heating, (c) ${ }^{\mathrm{Im}} \mathrm{TP}[\mathrm{La}]_{0.75}$ at $27^{\circ} \mathrm{C}$ on first heating, (d) ${ }^{{ }^{I m}} \mathrm{TP}[\mathrm{La}]_{0.5}$ at $27^{\circ} \mathrm{C}$ on first heating, (e) ${ }^{\mathrm{Im}} \mathrm{TP}[\mathrm{La}]_{0.75}$ at $27^{\circ} \mathrm{C}$ on first heating after thermal processing in a $10-\mathrm{T}$ magnetic field, and (f) ${ }^{\mathrm{Im}} \mathrm{TP}[\mathrm{La}]_{0.75}$ at $27^{\circ} \mathrm{C}$ after thermal processing in a 10-T magnetic field, followed by thermal processing in the absence of a magnetic field. Inserts are the corresponding POM images observed for (a-d) film samples on a glass substrate and (e, f) bulk samples $(\sim 5 \mathrm{mg})$ inside glass capillaries. Figure modified and reproduced from ref. (4) with permission from Springer Nature.

3b) on ${ }^{\mathrm{Im}} \mathrm{TP}[\mathrm{La}]_{075}$, a representative diamagnetic $\mathrm{LC}$ composite that adopts the Ortho structure at $27^{\circ} \mathrm{C}$ (Fig. 2c), revealed a dark POM image developed on cooling a birefringent texture under a 10-T magnetic field. Obviously, the assembling structures of ${ }^{\mathrm{Im}} \mathrm{TP}[\mathrm{La}]_{075}$ in the Ortho and $\mathrm{Col}_{h}$ II phases are optically anisotropic and display birefringent textures in POM, whereas that in the Cub phase is inherently optically isotropic to afford a dark POM image (Fig. 2; see the inserts). Thus, the in situ POM observations (Fig. 3b) in the presence of a 10-T field suggest that the induced Cub phase originates from the Ortho or $\mathrm{Col}_{h}{ }^{\text {II }}$ phase on cooling. To con- firm these observations, in situ XRD was used to monitor changes in the diagnostic diffraction peaks of ${ }^{\mathrm{Im}} \mathrm{TP}[\mathrm{La}]_{0.75}$ during thermal processing in a strong magnetic field $(7 \mathrm{~T})$. Before the application of a magnetic field, ${ }^{\mathrm{Im}} \mathrm{TP}[\mathrm{La}]_{0.75}$ showed broad peaks (Fig. 3c) assignable to reflections (110), (002), (200), and (111) of a Pbcm orthorhombic structure. These reflections disappeared on heating in the absence of a magnetic field, and a new broad peak emerged (Fig. 3d) due to the Ortho $\rightarrow \mathrm{Col}_{h}^{\text {II }}$ phase transition. Upon subsequent cooling in a 7-T magnetic field, strong diffraction appeared at $q$ $=1.96 \mathrm{~nm}^{-1}$, together with weak diffraction at $q=2.1$ 

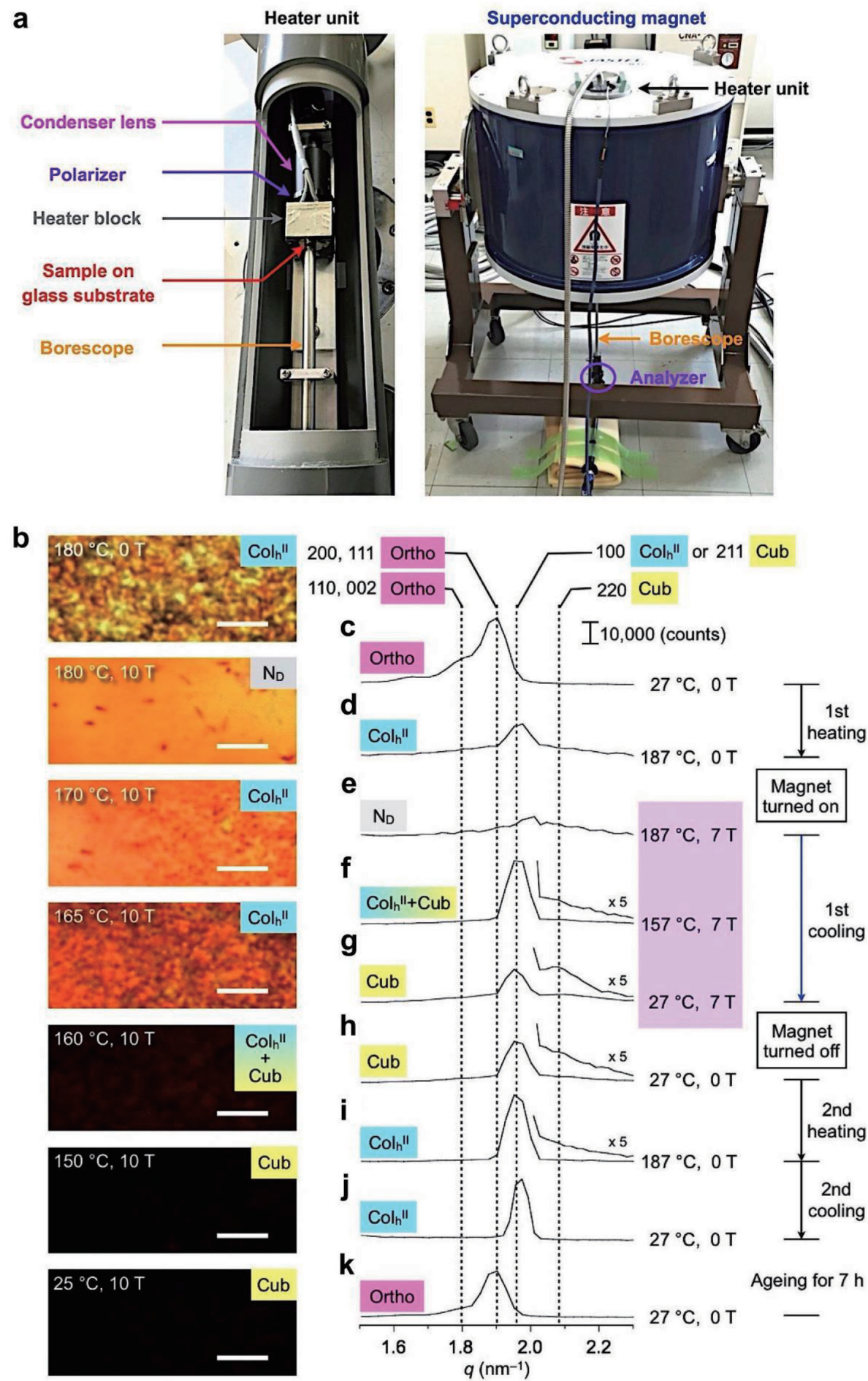

Fig. 3 (a) Experimental setup for in situ POM observation during thermal processing inside a 10-T magnet. (b) POM micrographs of a film sample of ${ }^{\mathrm{Im}} \mathrm{TP}[\mathrm{La}]_{0.75}$ on a glass substrate observed at $180^{\circ} \mathrm{C}$ (first heating; heating rate: $20^{\circ} \mathrm{C} / \mathrm{min}$ ) in the absence of a magnetic field, and observed at $180,170,165,160,150$, and $25^{\circ} \mathrm{C}$ upon cooling (cooling rate $\leq 0.5^{\circ} \mathrm{C} / \mathrm{min}$ ) in a 10-T magnetic field. Scale bars, $200 \mu \mathrm{m}$. The orange-colored POM image at $180^{\circ} \mathrm{C}$ and $10 \mathrm{~T}$ is due to the alignment of the $\mathrm{Col}_{h}^{\mathrm{II}}$ phase, which is accompanied by a thermal effect that induces a phase transition into a discotic nematic $\left(\mathrm{N}_{\mathrm{D}}\right)$ phase $^{4}$. (c-k) XRD patterns of a bulk sample of ${ }^{\mathrm{Im}} \mathrm{TP}[\mathrm{La}]_{0.75}$ in a glass capillary obtained (c, d) on first heating (heating rate: $20^{\circ} \mathrm{C} / \mathrm{min}$ ) in the absence of a magnetic field and $(\mathrm{e}-\mathrm{g})$ on first cooling (cooling rate: $0.5^{\circ} \mathrm{C} / \mathrm{min}$ ) in a $7-\mathrm{T}$ magnetic field, and obtained (h, i) on second heating (heating rate: $20^{\circ} \mathrm{C} / \mathrm{min}$ ) and $(\mathrm{j}, \mathrm{k})$ subsequent cooling (cooling rate: $0.5^{\circ} \mathrm{C} / \mathrm{min}$ ) in the absence of a magnetic field. In (j), due to the highly viscous nature of ${ }^{\mathrm{Im}} \mathrm{TP}[\mathrm{La}]_{0.75}$, the $\mathrm{Col}_{\mathrm{h}}{ }^{\mathrm{II}}$ phase was supercooled to $27^{\circ} \mathrm{C}$. Therefore, the measurement in (k) was conducted after aging ${ }^{I m} \mathrm{TP}[\mathrm{La}]_{0.75}$ for $7 \mathrm{~h}$ at $27^{\circ} \mathrm{C}$. Figure reproduced from ref. (4) with permission from Springer Nature. 
$\mathrm{nm}^{-1}$, resulting in a diffraction pattern ascribable to the coexistence of the $\mathrm{Cub}$ and $\mathrm{Col}_{\mathrm{h}}{ }^{\mathrm{II}}$ phases (Fig. 3f). On further cooling to $27^{\circ} \mathrm{C}$ under a 7 - $\mathrm{T}$ field, the intensity of the strong peak decreased, whereas that of the weaker increased, resulting in two reflections with intensity ratio and $q$-spacing ratio that agreed well with the (211) and (220) reflections of an Ia3d cubic structure (Fig. 3g). These induced diffraction peaks persisted stably at $27^{\circ} \mathrm{C}$ even under zero applied magnetic field (Fig. 3h). However, in a second heating and cooling cycle in the absence of a magnetic field, the system recovered the diffractions characteristic of the original Ortho phase (Fig. 3k). Therefore, these in situ XRD results, which are consistent with the in situ POM observations (Fig. 3b), indicate that the Cub phase of ${ }^{\mathrm{Im}} \mathrm{TP}[\mathrm{La}]_{0.75}$ starts to emerge below the clearing point from the Col${ }_{\mathrm{h}}^{\mathrm{II}}$ phase at $\sim 160^{\circ} \mathrm{C}$ under a 7 - $\mathrm{T}$ field, and then develops over the entire material at $27^{\circ} \mathrm{C}$.

To gain further understanding of the physical mechanism of this magneto-assisted phase selection, Hajjaj et $a l$. evaluated the effect of a magnetic field on the free energy $\left(E_{\mathrm{mag}}\right)$ of the diamagnetic LC composite using the equation $E_{\mathrm{mag}}=-\chi B^{2} / 2 \mu_{0}$, where $\chi$ is the mass magnetic susceptibility, $B$ is the magnetic flux density, and $\mu_{0}$ is the magnetic permeability of vacuum ${ }^{4,9,15)}$. Here, $\chi$ values were determined by a superconducting quantum interference device (SQUID), at a temperature $\left(27^{\circ} \mathrm{C}\right)$ and magnetic field intensity $(0.5 \mathrm{~T})$ where neither phase transition nor alignment takes place in ${ }^{\mathrm{Im}} \mathrm{TP}[\mathrm{La}]_{x}$ composite $^{4)}$. Based on SQUID measurements, the estimated $E_{\text {mag }}$ value is significantly higher for the Ortho phase compared to the other LC phases of ${ }^{\operatorname{Im}} \mathrm{TP}[\mathrm{La}]_{x}$ (Fig. 1b), and therefore the Ortho phase should be more destabilized by magnetic field interactions.

All these experiments confirmed the occurrence of a magneto-assisted phase selection in the LC assembly, whereby the Ortho phase can be reprogrammed into the Cub phase by diamagnetic interactions. This unusual and memorized behavior is promising for nonvolatile memory device applications. Here, for example, the structural information of the LC material can be written and memorized by an applied magnetic field and then erased by heating (e.g., laser pulse) without field application. In analogy to the conventional thermotropic and lyotropic LCs that exhibit a phase transition in re- sponse to thermal stimuli and the presence of solvents, respectively, the magneto-assisted phase selection in LC materials can be referred to as magnetotropic. This exotic phenomenon, widely believed to be impossible, advances our understanding of the interplay between magnetic fields and organic matter and ultimately provides a design strategy for advanced responsive systems.

\section{PDLC Composite Films: From Device Technology to Functional Cosmetics}

\subsection{The human skin beyond the biological definition}

The skin provides the body with protection from infection and the environment, as well as sensory capabilities. Skin is also a visible interface, with its appearance reflecting vigor or barrier dysfunction, leaving those with skin problems, such as aging and dryness, vulnerable to detrimental psychological effects ${ }^{16)}$. In this context, methods to reproducing the functional and aesthetic properties of healthy human skin would be of great interest. From a materials science perspective, the human skin is a highly complex soft composite system. However, its essential features are reproducible using wearable sensory and protective interfaces. The former interfaces are realizable by electronic skin (eskin), such as those developed by Someya's group, where millions of sensors are embedded in a flexible polymer film to communicate with a large skin surface area $^{17)}$. Such wearables can also contain sensors to monitor physiological signals, such as calcium ion concentration $^{18)}$ that is a diagnostic indicator of the skin barrier condition ${ }^{19)}$, and therefore hold a great promise as skin-monitoring gadgets and unconventional cosmetics. As protective interfaces, topically applied polymer films, known as second skins, are innovative cosmetic solutions to many skin problems. These functional films can serve as a physical barrier to environmental insults and cutaneous water loss while imparting an aesthetic appearance to the skin. Building on the concept of skinlike wearable, Yu et al. synthesized a crosslinked polymer layer (XPL), which is engineered with specific elasticity and tensile strength to reproduce the elastic response in normal youthful skin ${ }^{20)}$. The XPL film forms in situ on the skin, following the topical application of a flowable mixture of fumed silica and reactive polysiloxane that is then crosslinked by a platinum catalyst. 
This elastic second skin is invisible, moisturizing, safe, and provides enhanced mechanical integrity to the underlying skin. Another promising approach for the industrial production of high-performance second skins is the fine-fiber technology or the electrospinning of polymer solutions ${ }^{21)}$. Although these approaches and others provided the second skins as topical cosmetic formulations, their polymer processing technologies are harsh for biopolymers and not compatible with the barrier lipids. Namely, ceramides and sterols, which usually crystallize once concentrated in the condensed film state. Because these lipids are essential components of the skin barrier function ${ }^{22,23)}$, including them in the second skin is highly desirable.

Ideal second skins should be designed to mimic the structure and functionality of the stratum corneum (SC), the outermost skin layer that provides the primary barrier function of the $\operatorname{skin}^{22,23)}$. In the SC, barrier lipids such as ceramides, free fatty acids, and cholesterol are densely packed and form a continuous multilayered structure (i.e., lamellar lipid sheets) with an LClike nature, which plays an essential role in the formation of the skin barrier ${ }^{22,23)}$. Such multilayered (multilamellar) assembly of lipids in condensed states is realizable by substrate-assisted methods, such as the layer-by-layer approach reported by Leal's group ${ }^{24,25)}$. Recent advances in this field have led to composite polymer and lipid films with intriguing biological functionalities $^{24,25)}$. However, their commercial applications have been hampered due to substrate use. To address the design challenges of a wearable lamellar lipid film, Hajjaj et al. used the PDLC technology to stabilize LC microdroplet dispersions, enriched with lipids, in polymeric films ${ }^{26)}$. PDLC technology has been extensively exploited to produce highly functional composite films, mainly for device applications ${ }^{5)}$. Notably, lamellar lipids or lyotropic LC phases have never been considered before for a cosmetic PDLC film. Most likely, due to the general belief that lamellar lipids cannot exert their functions, such as barrier repair and delivery, without permeating the skin surface ${ }^{6,27,28)}$. Accordingly, cosmetic formulations of the SC lamellar lipids are limited to conventional technologies that would allow their intake by topical applications, such as lamellar gels, liposomes, or even liposome-polymer composite films.

Hajjaj et al. serendipitously found that the immobilization of a large number of lamellar lipids onto the skin, in a manner to form a wearable PDLC film when topically applied, served as a second skin barrier. Based on experimental and clinical evidence, the prepared

Table 1 Ingredients (wt\%) used in the formulation of PDLC films and control polymer films. Reproduced from ref. (26) with permission from The Society of Cosmetic Chemists of Japan.

\begin{tabular}{|l|c|c|c|c|}
\hline \multicolumn{1}{|c|}{ Ingredient } & PDLC $1^{\mathrm{a}}$ & Control 1 & PDLC 2 & Control 2 \\
\hline Lecithin $^{\mathrm{b}}$ & 1.10 & 0 & 1.10 & 0 \\
\hline Stearic acid & 0.40 & 0 & 0.40 & 0 \\
\hline Phytosterol & 0.50 & 0 & 0.50 & 0 \\
\hline Glycerol & 2.00 & 2.00 & 2.00 & 2.00 \\
\hline BG & 2.00 & 2.00 & 2.00 & 2.00 \\
\hline HPMC & 2.00 & 2.00 & 0 & 0 \\
\hline Sodium HA & 0.40 & 0.40 & 0 & 0 \\
\hline PVA & 0 & 0 & 1.00 & 1.00 \\
\hline Acrylate & 0 & 0 & 1.00 & 1.00 \\
\hline L-arginine & 0 & 0 & 0.96 & 0.96 \\
\hline Trehalose & 1.00 & 1.00 & 0 & 0 \\
\hline Phenoxyethanol & 0.12 & 0.12 & 0.12 & 0.12 \\
\hline Methylparaben & 0.10 & 0.10 & 0.10 & 0.10 \\
\hline Water & 90.38 & 92.38 & 90.82 & 92.82 \\
\hline
\end{tabular}

${ }^{a}$ Alternatively, ceramide VI can be used instead of lecithin (PDLC ceramide).

${ }^{\mathrm{b}}$ Phosphatidylcholine (PC) content in the range of $65-75 \%$.

${ }^{c}$ Neutralized with L-arginine (pH: 6.00). 
PDLC cosmetic film demonstrates features reminiscent of human SC, namely those related to the lamellar lipid assemblies, barrier function, surface structure, and water retention behavior ${ }^{26)}$.

\subsection{Preparation and characterization of cosmetic PDLC films}

For the preparation of the LC component in the cosmetic PDLC film, Hajjaj et al. emulsified a solid premix of phytosterol (25 wt\%), stearic acid (20 wt\%), and lecithin or ceramide (55 wt\%) in a 1:1 mixture of glycerol and 1,3-butylene glycol $(\mathrm{BG})^{26)}$. The emulsified lipid mixture (i.e., lyotropic LC phase) was then dispersed in an aqueous phase containing water and the following ingredients (Table 1): osmolyte (trehalose), preservatives (phenoxyethanol and methylparaben), and film-forming polymers. Here, a combination of biopolymers, such as sodium hyaluronate (sodium HA) and hydroxypropyl methylcellulose (HPMC), or synthetic polymers, such as acrylates/C10-30 alkyl acrylate cross-polymer (acrylate) and polyvinyl alcohol (PVA), served as a hydrogel matrix. The polymers' selection is dependent on their ability to provide steric and osmotic stabilizations to the dispersed lyotropic LC phase ${ }^{29)}$. Under the high dilution condition of the emulsion state (water content $\sim 90$
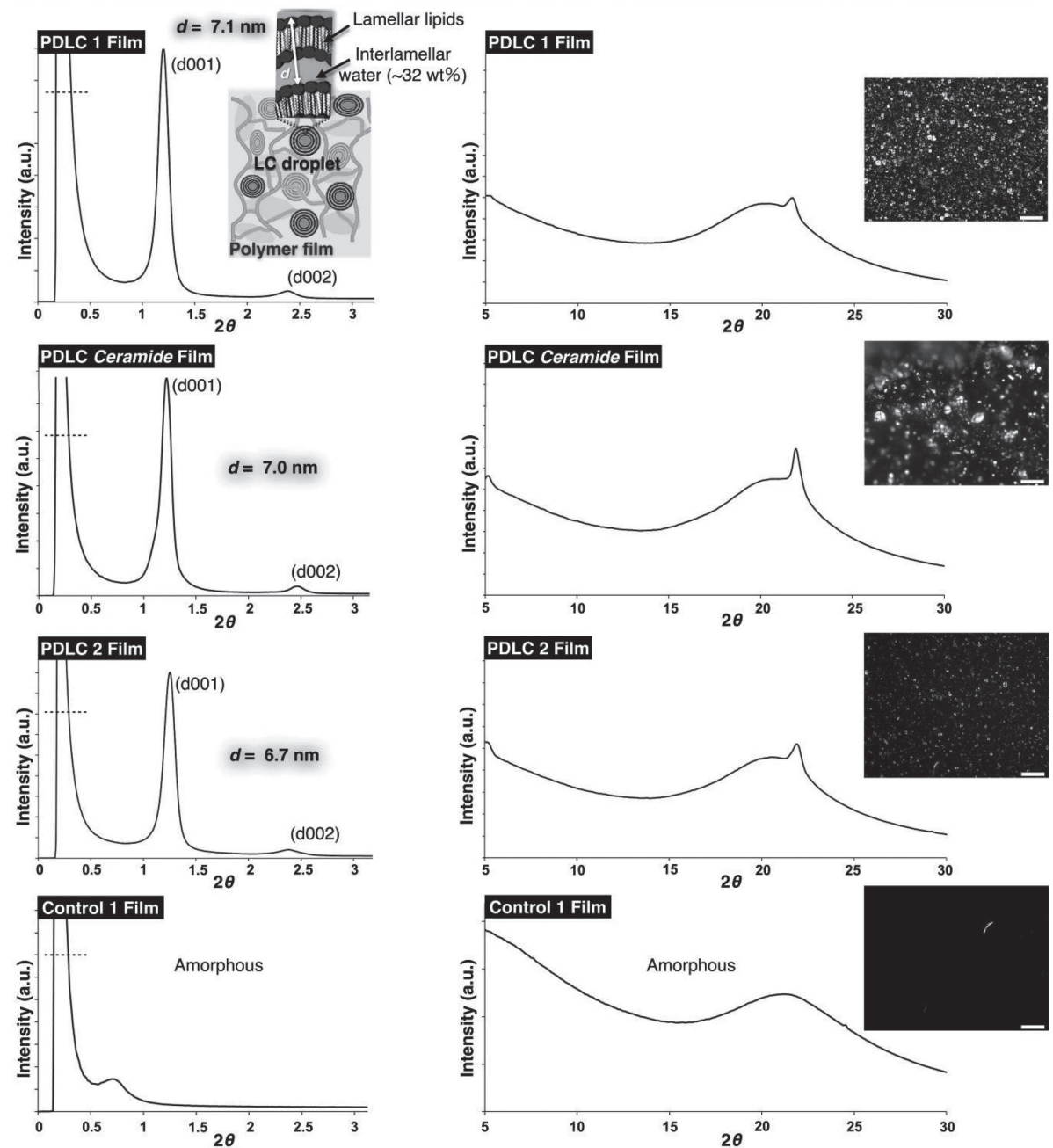

Fig. 4. XRD structural characterizations of PDLC films and a control polymer film at $25^{\circ} \mathrm{C}$. The film samples were obtained after $5 \mathrm{~h}$ of topical application. Left panel: small-angle XRD patterns, which confirmed the formation of a lamellar lipid organization in the prepared PDLC films. The schematic insert depicts the interlamellar water contained in PDLC 1 film. Right panel: wide-angle XRD patterns, where the broad peak $\left(2 \theta \sim 19.8^{\circ} ; d=0.45 \mathrm{~nm}\right)$ is due to the liquid-like arrangement of the lipid side chains in the lamellar phase, and the overlapped sharper peak $\left(2 \theta \sim 21.6^{\circ} ; d=0.41 \mathrm{~nm}\right)$ is a consequence of the polymer film's osmotic stress, which dehydrates the lamellar bilayers and consequently forces the lipids to pack densely in a gel-like state ${ }^{30}$. This peak diminishes at human skin temperature $\left(32-36^{\circ} \mathrm{C}\right)$. Inserts are the corresponding POM images observed for the film samples at $25^{\circ} \mathrm{C}$. Here, Control $1 \mathrm{film}$ appears dark (isotropic), whereas the PDLC films show birefringent microdroplets (Maltese crosses) due to the dispersed LC phase. Scale bars, $50 \mu \mathrm{m}$. Figure modified and reproduced from ref. (26) with permission from The Society of Cosmetic Chemists of Japan. 
wt\%), the amphiphilic lipids self-assembled into a nonlamellar structure (i.e., intermediate rectangular phase), as confirmed by small-angle X-ray scattering (SAXS) measurement ${ }^{29)}$. Notably, the obtained PDLC emulsions (Table 1) are very stable on storage at $25^{\circ} \mathrm{C}$ for $\sim 3$ years, where the osmotic stress of the polymer hydrogel matrix governs their stabilities. Most likely, because the polymer-LC interaction in the PDLC emulsions is physical rather than chemical in nature, a known feature for the PDLC systems ${ }^{5}$.

When the PDLC emulsions are applied onto the skin and allowed to dry, transparent PDLC films are obtained. The PDLC films are skin-adherent and can be removed from the skin surface as free-standing sheets, which allowed their characterizations as second skins. Figure 4 shows the optical and structural characterizations of representative PDLC films and a control polymer film obtained after $5 \mathrm{~h}$ of topical application. Interestingly, the lipid assembly in the PDLC films exhibited structural features reminiscent of the lipid organization in the SC. As evidenced by the XRD analysis (Fig. 4; left panel), the lipids formed a lamellar structure in the condensed film state with a periodicity $(d)$ of $\sim 7 \mathrm{~nm}$, which may resemble the lamellar lipid organization in human SC, namely, the short periodicity phase (SPP) with $d \sim 6 \mathrm{~nm}^{22,23)}$. Besides the lamellar lipid organization, the wide-angle XRD analysis of the PDLC films (Fig. 4; right panel) revealed the predominant presence of a disordered lipid phase (LC phase; $d \sim 0.45 \mathrm{~nm}$ ) coexisting with an ordered lipid phase (gel phase; $d \sim 0.41$ $\mathrm{nm})$. Although crystalline lipid phases predominately exist in human SC, XRD measurements on model mixtures of SC lipids indicated the formation of ordered orthorhombic $(d \sim 0.37 \mathrm{~nm})$ and hexagonal $(d \sim 0.41 \mathrm{~nm})$ lipid phases along with a disordered lipid phase (LC; $d$ $\sim 0.46 \mathrm{~nm})^{22,23)}$.

The fact that the PDLC film readily forms on human skin as a lamellar lipid-sheet, without using a dedicated applicator or any substrate, suggests that its preparation is feasible on an industrial scale ${ }^{29)}$.

Hajjaj et al. used a combination of DSC and thermogravimetric analysis (TGA) to investigate the thermal phase behavior and water retention properties of the PDLC films ${ }^{26)}$. Figure 5 shows the DSC profiles of a PDLC film, control polymer film, and hydrated SC sheet taken from the back of a healthy subject (35 years). The DSC and TGA results indicated that water is better retained in the PDLC film than in the control polymer film (Fig. 5), through the interactions with the hydrophilic groups of both the polymers and lipids. Be-
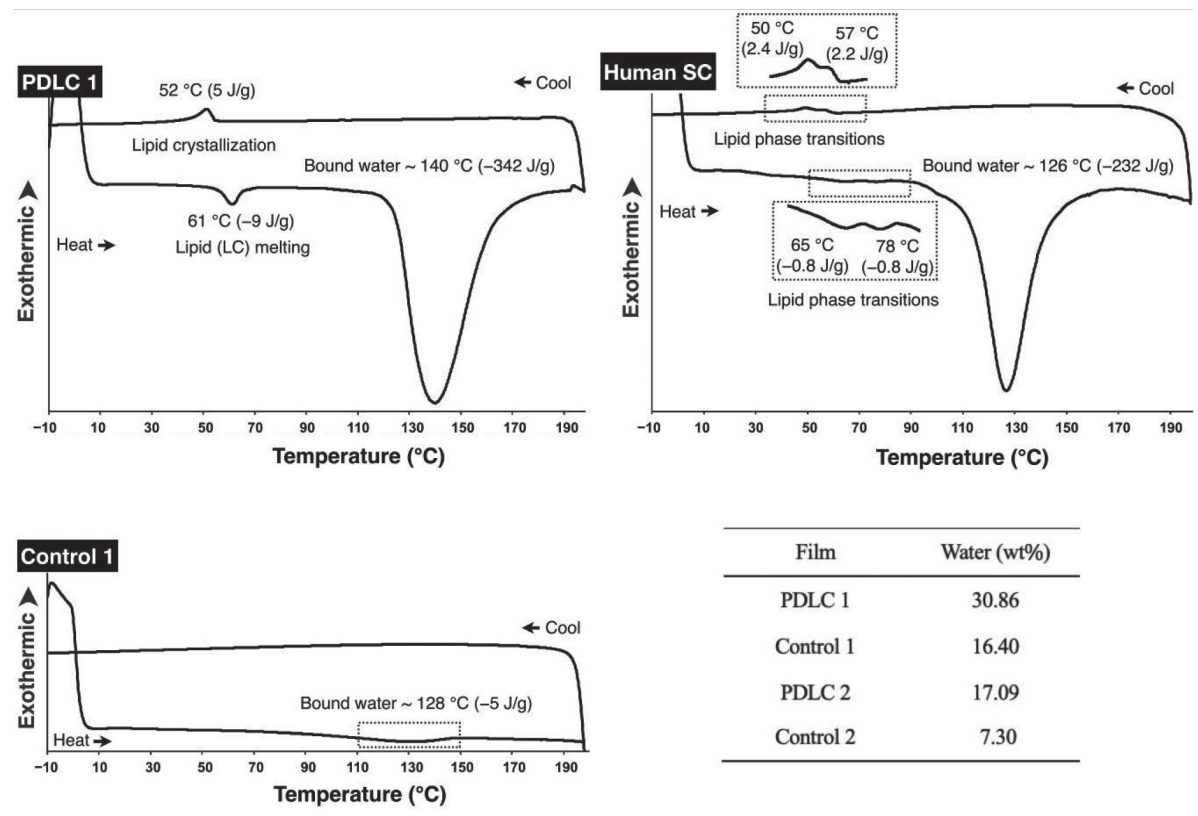

\begin{tabular}{cc}
\hline Film & Water (wt\%) \\
\hline PDLC 1 & 30.86 \\
Control 1 & 16.40 \\
PDLC 2 & 17.09 \\
Control 2 & 7.30 \\
\hline
\end{tabular}

Fig. 5 DSC profiles (first heating/cooling cycle) of representative PDLC and control polymer films as well as a hydrated SC sheet, measured at a scan rate of $5{ }^{\circ} \mathrm{C} / \mathrm{min}$. The film samples were obtained after $5 \mathrm{~h}$ of topical application. Values in parentheses represent the change in enthalpy $(\Delta \mathrm{H})$. The average water content (wt\%) in the film samples $(n=6)$ was determined by DSC-TGA simultaneous thermal analysis. Figure reproduced from ref. (26) with permission from The Society of Cosmetic Chemists of Japan. 
cause of the excellent moisture retention properties of biopolymers, PDLC 1 film retained more water than PDLC 2 film (Fig. 5) of the synthetic polymer series. Notably, the DSC profile of the PDLC film showed the essential features of a hydrated human SC (Fig. 5), namely those related to the lipid phase transitions ${ }^{31)}$ and evaporative water loss induced by heating. Even more remarkable is the fact that the water content of the PDLC film is in the range of that reported for a healthy human SC ( 20-30 wt $\%)^{32)}$.

The most striking feature of the lipid (LC) inclusion in polymer film is the unique surface structure, revealed by atomic force microscopy $(\mathrm{AFM})^{26)}$. As shown (Fig. 6a), the AFM images observed for the control polymer film show a very rough surface morphology. This morphology may be caused by the deformation of the hydrophilic polymer film due to unfavorable inter- actions with the hydrophobic substrate surface. To alleviate the energy of unfavorable interactions with the substrate, the polymer chains in the PDLC film should undergo a structural deformation that partially exposes the hydrophobic LC microdroplets to the interfaces. This is supported by AFM observations on PDLC 1 film surface (Fig. 6b), where a spherical soft domain (LC) with a considerable height mismatch with the background (polymer film) appeared exposed at the surface. Such topographical features would energetically allow for a larger contact area with the substrate (i.e., smooth surface formation), thereby improving the polymer film adhesion to hydrophobic surfaces. These results corroborated the water contact angle (WCA) measurements, where PDLC 1 film surface showed a more hydrophobic character (WCA $\sim 45^{\circ}$ ) compared to Control 1 film surface $\left(\mathrm{WCA} \sim 10^{\circ}\right.$ ). Notably, PDLC 1 film

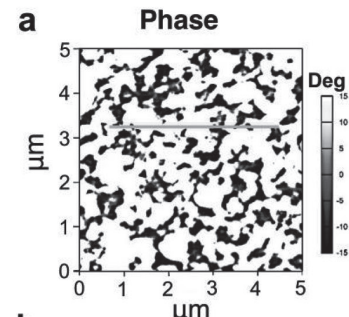

b
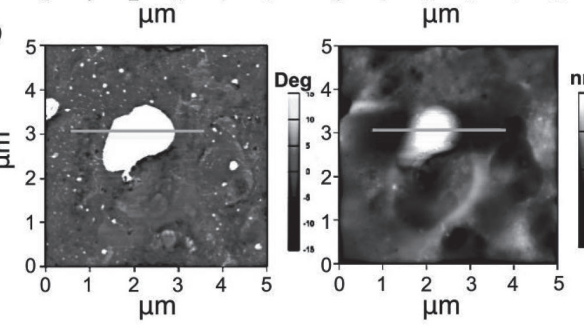

Control 1

C
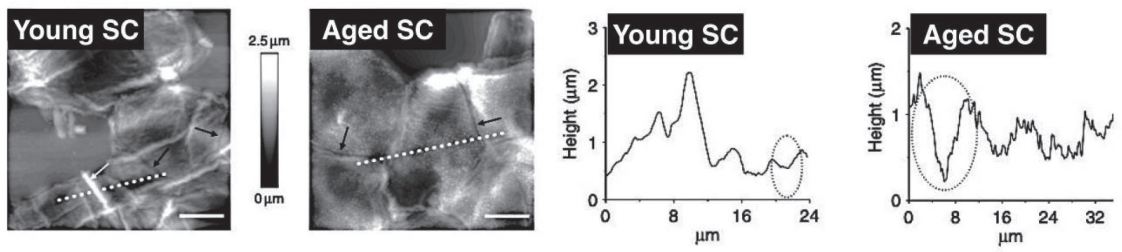

Fig. 6 (a, b) AFM images (scan size: $5 \mu \mathrm{m} \times 5 \mu \mathrm{m}$ ) and height profiles of representative film samples supported on a polyurethane surface. (a) Phase and topographic images, along with the height profile of the marked line, of Control 1 film. Due to its hydrophilic nature, Control 1 film assumed a very rough surface structure to minimize the unfavorable interactions with the hydrophobic substrate surface. (b) Phase and topographic images, along with the height profile of the marked line, of PDLC 1 film. The phase separation in the PDLC 1 film resulted in two domains: lighter (soft) spheres and darker (hard) background. The spherical domain (microfeatures) is due to the dispersed LC microdroplets in the polymer film. Figure (a, b) reproduced from ref. (26) with permission from The Society of Cosmetic Chemists of Japan. (c) AFM images and height profiles of a native SC (layers of corneocytes) tape-stripped from young (39 years) and aged (70 years) donors. The dotted line indicates a cross-section of the corneocyte layer displayed in the height profile. The white arrow points to an unidentified filamentous structure and the black arrows indicate the cell-cell contact of two corneocytes, with a discrete (young) or prominent (aged) intercellular gap (dotted circle in the height profile). The smooth areas in the AFM images are the tape surface, and the fluctuations in the height profile of the aged SC are due to increased cell surface roughness. Scale bars, $10 \mu \mathrm{m}$. Figure (c) reproduced from ref. (33) with permission from the author and John Wiley and Sons. 
surface (Fig. 6b) showed a smooth microfeature (LC droplet; lateral dimension $\sim 2 \mu \mathrm{m}$ ) which is reminiscent of that observed by Schneider and coworkers for young skin $^{33)}$. Here, AFM observations on young SC (Fig. 6c) revealed a smooth microfeature (corneocyte; lateral dimension $\sim 2 \mu \mathrm{m}$ ) with a barely visible intercellular gap. Such a topographical feature was not observed for aged SC (Fig. 6c), which showed a very rough microfeature (corneocyte; lateral dimension $\sim 24 \mu \mathrm{m}$ ) with a prominent intercellular gap ${ }^{33}$. These unique surface features of the PDLC film are reflected in the macroscopic properties. For example, Hajjaj et al. showed that PDLC 1 film is an effective barrier against organic micropollutants, with exceptional antipollution performance that surpassed those of Control 1 film and a skin model $^{26)}$. Furthermore, the in vivo adhesion (vide infra) was also greatly influenced by the nature of the PDLC film surface ${ }^{26)}$.

\subsection{PDLC beauty film as a safe and effective second skin barrier}

Based on in vitro studies, Hajjaj et al. demonstrated that the PDLC film is a diffusion barrier to water vapor flux (i.e., breathable barrier), while it effectively suppressed evaporative water loss at skin surface temperature $\left(\sim 32^{\circ} \mathrm{C}\right)^{26)}$. This exceptional occlusivity, which is significantly higher than that of the control polymer

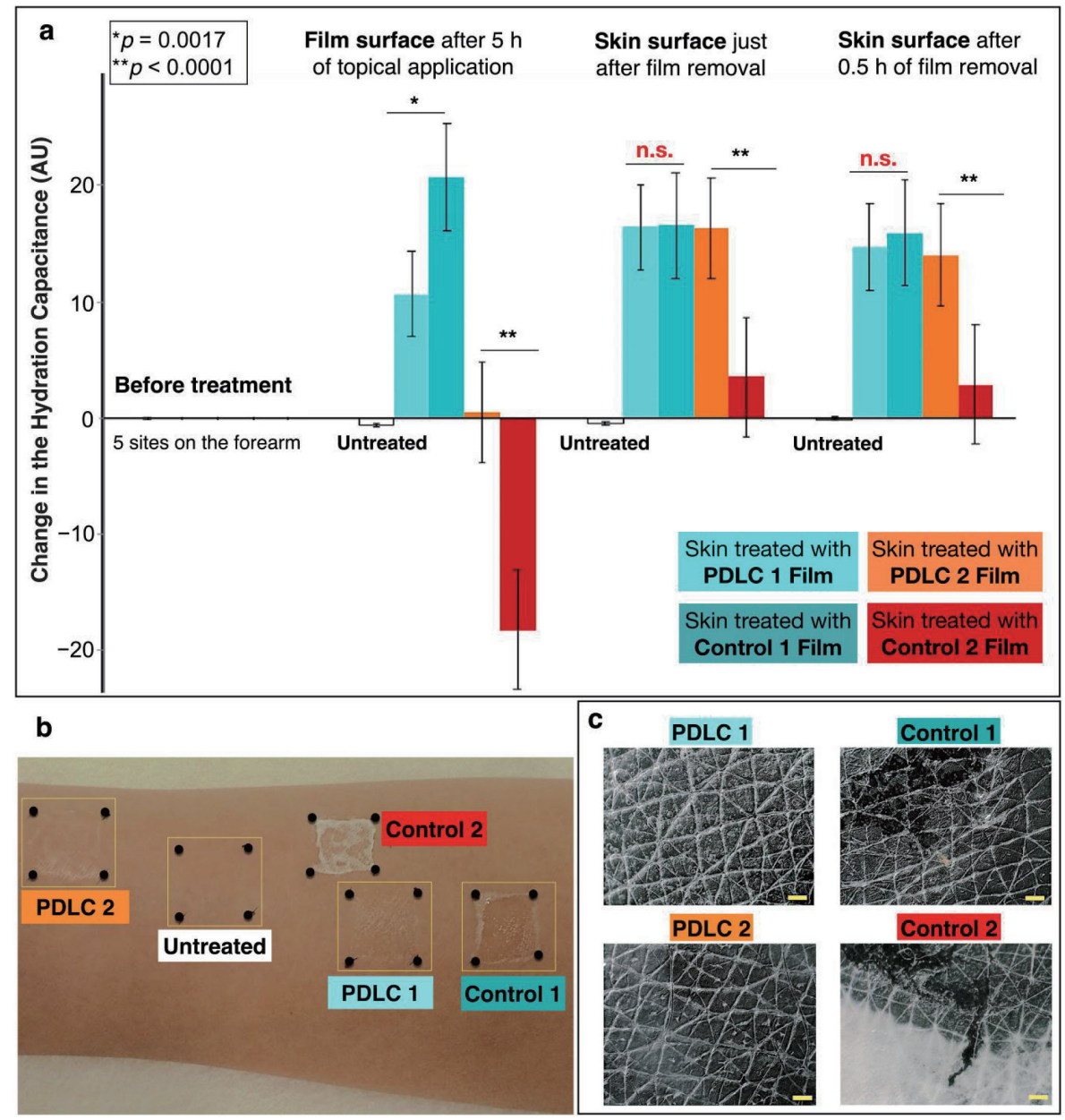

Fig. 7 (a-c) Human study $(n=10)$ evaluating the performance of representative PDLC and control films after $5 \mathrm{~h}$ of topical application. (a) Induced changes in the skin hydration evaluated as changes in the skin capacitance relative to baseline. The error bars represent the standard errors of the mean. Data sets marked with asterisks represent statistically significant differences using a two-sided $t$-test. AU: arbitrary unit; n. s.: not significant. (b) A subject forearm while wearing the tested films, following $5 \mathrm{~h}$ of topical application. Due to shrinkage, Control 1 film peeled off spontaneously from the edges and Control 2 film developed large open cracks. Note that PDLC 1 and PDLC 2 films can remain adhered to the skin surface as a continuous sheet for more than 8 h. (c) Digital images of the films which were tape-stripped from the subject's forearm in (b). Contrary to the control films, PDLC 1 and PDLC 2 films did not disintegrate and were removed from the skin surface as a continuous sheet. The images were acquired by a laser scanning confocal microscope. Scale bars, $0.10 \mathrm{~mm}$. Figure reproduced from ref. (26) with permission from The Society of Cosmetic Chemists of Japan. 
film, is due to the high tortuosity of the PDLC film, where the hydrophobic LC microdroplets suppress the water vapor transmission in the hydrophilic polymer matrix. The skin compatibility of the PDLC film was further elucidated in vivo on 10 subjects $(n=10$; average age $=41.07$ years), where its excellent clinical performance (Fig. 7) is reasoned to result from a unique hydration mechanism involving regulation of the polymer film water content by the LC component ${ }^{26)}$. Such skin hydration mechanism was more evident for the bio-based films (PDLC 1 vs Control 1; Fig. 7a), as a result of the higher amount of water retained in the biopolymer matrix. For the synthetic polymer films (PDLC 2 vs Control 2; Fig. 7a), the in vitro occlusion performances were reflected in vivo, and therefore, the skin hydration resulting from wearing these films is more likely due to the occlusion effect (i.e., conventional occlusive hydration). Although PDLC 1 film and Control 1 film hydrated the skin to the same extent (Fig. 7a; skin surface), the absence of a regulatory mechanism for water transfer in Control 1 film resulted in a compromised barrier performance (Fig. 7b, c). This is because the loosely-bound excess water in Control 1 film is more likely to be exchanged at the interfaces, thereby causing a wet surface condition at the air interface (Fig. 7a; film surface) and instant hydration of the underlying skin (Fig. 7a; skin surface). Consequently, following the $5 \mathrm{~h}$ application period, Control 1 film dried (Fig. 5) and disintegrated on the skin surface (Fig. 7b, c). The clinical performance of the polymer film was dramatically improved by the inclusion of the LC component, hinting at a regulatory role of the lamellar LC in the PDLC 1 film hydration mechanism (Fig. 8). This is supported by the XRD observation that the interlamellar water content in PDLC 1 film (Fig. 4; schematic insert) gradually decreases during the $5 \mathrm{~h}$ application period. Here, during deswelling and dehydration on the skin surface, the hydrophilic polymer component of PDLC 1 film osmotically absorbs water from the LC phase, thereby inducing a reduction in its lamellar spacing. This implies that the lamellar LC phase is indirectly involved in the skin hydration resulting from PDLC 1 film wear, probably by supplying moisture to the polymeric film that primarily hydrates the skin. In contrast, the LC phase in PDLC 2 film showed only a slight decrease in its lamellar spacing during the $5 \mathrm{~h}$ application period and therefore was assumed to be less involved in the skin hydration resulting from PDLC 2 film wear. Besides regulating the water content in the polymer film through osmosis, the lamellar LC phase $(d \sim 7 \mathrm{~nm})$ also restricts water vapor diffusion in PDLC 1 film through the tortuosity effect, ultimately resulting in a safe occlusion and adhesion in vivo (Fig. 8). Interestingly, even in human skin, the intercellular lamellar lipids (SPP; $d \sim 6 \mathrm{~nm}$ ) were reported to be involved in regulating the water content in the $\mathrm{SC}^{32}$.

Remarkably, besides improving the polymer in vivo hydration and adhesion performances, the LC component in the PDLC film also acted as an optical modifier. As shown in Figure 7 (b), the optical properties of the polymeric films, such as gloss and transparency, were greatly modulated in the PDLC film to a degree that matched the underlying skin, thereby complementing the aesthetic appearance of the film on the skin. Of note, a previous approach for preparing a second skin relied on light scattering particles to tune the polymer optical properties with the light-scattering properties of natural skin ${ }^{20)}$. Although the cosmetic appearance was improved, the used optical modifiers cause limitations to scalable production. Noteworthy, the droplet size of the LC dispersion is critical to the overall performance of the PDLC film on the skin, where submicron dispersions of the lipids (i.e., liposomes) afforded a composite film with features that are virtually similar to those of the control polymer films. Therefore, PDLC film should not be confused with the liposome-polymer composite film, where the confined micron-sized LC droplets ( 1-10 $\mu \mathrm{m})$ are the main features of the PDLC films, which are essential to modulate the polymer film's properties $^{5)}$.

Taken together, the wearable functional cosmetic prepared as a PDLC film is unprecedented, where the immobilization of a high condensation of lamellar lipids onto the skin by soft interface served as mimicry of the SC and acted as a second skin barrier. In analogy to the device PDLC films which operate under temperature or electric field gradients ${ }^{5}$, the beauty PDLC film may function in vivo under a water content gradient (Fig. 8), hence rendering such occlusive film safe, moisturizing, well-tolerated, and convenient for prolonged wear as a second skin ${ }^{26)}$. 

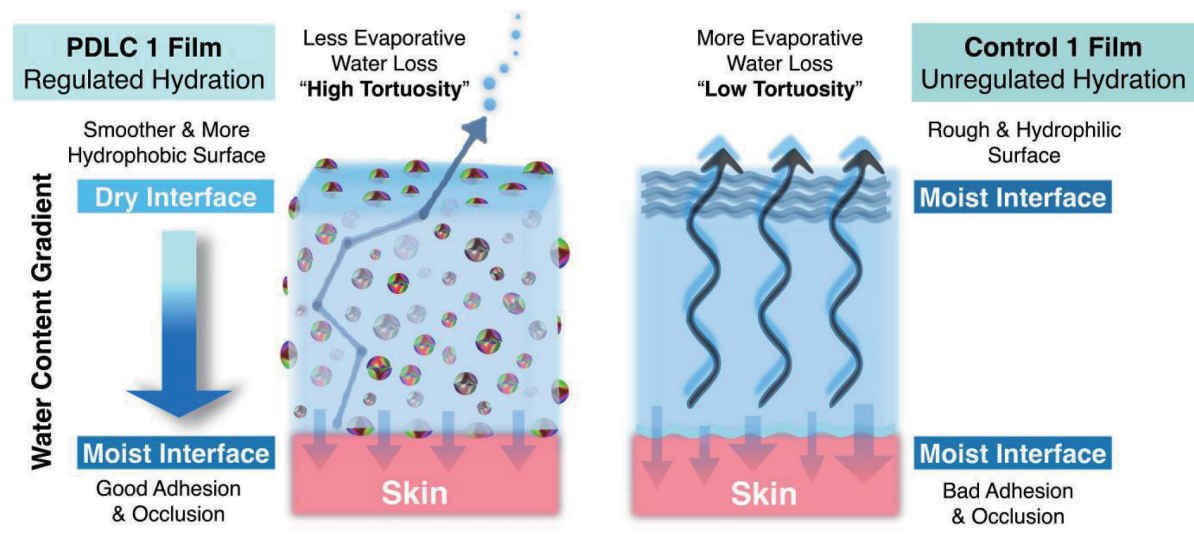

Fig. 8 A proposed mechanism for PDLC 1 film hydration in vivo. Without the lamellar lipid (LC microdroplets) the hydration of the polymer film is not regulated, resulting in relatively wet interfaces that compromise the barrier performance of Control 1 film on the skin. The inclusion of the LC component dramatically improved the barrier performance of the polymer film on the skin. This is because the lamellar LC regulates the water flux through osmosis and tortuosity effects, thereby generating a water concentration gradient across the PDLC 1 film that is crucial for its performance. The indicated hydration states (Dry, Moist) at the film interfaces are based on the results of a preliminary in vivo study using confocal Raman spectroscopy. The partially exposed LC droplets at the PDLC 1 film interfaces (see Fig. 6b) are believed to facilitate the adhesion, by acting as anchor points between the polymer film and skin surface. Figure reproduced from ref. (26) with permission from The Society of Cosmetic Chemists of Japan.

\section{Conclusions and Outlook}

Magnetic fields are generally known to interact with organic matter non-destructively, leaving its molecular structure unaffected, even when a strong magnetic field generated from a superconducting magnet is applied. Accordingly, most research for all diamagnetic materials has been focused on using strong magnetic fields to induce alignments or shape changes, which make such diamagnets anisotropic and functional. The magneto-induced structural selection was considered impossible for diamagnets, and if it occurs, it should bring about fascinating emergent behavior and exotic phenomena as it involves changes in the material's molecular structure. In principle, LC composite can be engineered to promote the formation of metastable or intermediate phases, such as the Ortho phase of ${ }^{\mathrm{Im}} \mathrm{TP}[\mathrm{La}]_{x}$ composite. Although thermodynamically stable, such diamagnetic intermediate states can show unusual phase behavior when exposed to strong magnetic fields. The observation that a diamagnetic LC composite selectively forms different structures with distinct properties in response to magnetic field interactions is unprecedented, which updated the widely held belief that a strong magnetic field cannot significantly alter the fundamental structure of a diamagnetic molecular assembly. Such unprecedented phase behavior of a diamagnetic molecular assembly, referred to as "magneto- tropic" LC, should have a profound impact in various fields of science and substantially expand our understanding of the interplay between organic matter and magnetic fields. Since most materials are diamagnetics, exploring strong or even extreme field interactions is essential for creating new functionality, which can lead to advanced materials and devices that revolutionize the industry. However, this requires dedicated in situ techniques for monitoring the diamagnet behavior under strong or extreme magnetic fields, which allows for trapping the transiently induced states to ambient conditions. Besides the development of novel experimental tools, finding microscopic explanations for the macroscopically observed behavior in LC is another grand challenge that might be addressed by theory and simulations.

Recent advances in polymer chemistry and materials science have enabled researchers to come close to mimicking the skin's properties, such as its sensing capabilities, elastic recoil, and even its healthy appearance. Topically-applied polymer films or second skins, which mimic youthful skin properties, are innovative cosmetic solutions to many skin problems. Existing polymer processing technologies have provided impressive second skins as topical cosmetic products, but they are usually harsh for biopolymers and not compatible with SC lipids, the key components of the cutaneous barrier. By considering the current trend in the cos- 
metic industry, driven by the increasing consumers' demand for natural non-synthetic ingredients, biopolymers are better alternatives for the second skin. Unfortunately, biopolymers cannot serve as a wearable barrier due to their inherent hydrophilic nature with mechanical and optical properties that do not match the skin. PDLC is a practical technology to engineer specific adhesion, occlusivity, transparency, surface structure, water content, and other desirable barrier properties to biopolymers. This device technology enables the confinement of millions of LC microdroplets (i.e., SC lipids) in a condensed biopolymer matrix, affording a functional wearable barrier compatible with the skin. With such lipid feature embedded in it, the bio-based PDLC film may serve as the next generation of a skin-inspired wearable barrier. Further research will explore possibilities for engineering responsiveness into the PDLC film, exploiting the skin-like adaptive nature of the biopolymers optimized by the LC component. Beyond mimicking the SC properties, the future design of bio-based second skins should also consider improving the skin's normal protection capabilities, for example, against harmful sun exposure. Indeed, the melanin/keratin composite recently developed by Nowogrodski et al. will allow for desirable sun protection and skin color for the bio-based second skins, which may ultimately enable them to serve as unconventional sunscreens ${ }^{34)}$

A further implication of the reviewed findings is the possibility of using magnetic fields as a noninvasive methodology to address the skin barrier dysfunction. A preliminary study has shown that a weak magnetic field $(<1 \mathrm{~T})$ can influence the lipid order in a native biological membrane through a diamagnetic response ${ }^{35}$. Here, enhanced lipid order (i.e., improved barrier performance) was observed during magnetic field interactions with the membrane structure, resulting in an increase in the gel-ordered phase and a decrease in the liquid-disordered phase of the lipids. If such an effect occurs in the intercellular lipids in human SC, then it would be possible to treat many skin problems noninvasively, as magnetic field interactions would remotely stimulate a reparative effect on the compromised skin barrier. Such a scenario is not impossible, considering that the skin is a soft diamagnetic composite that might show a response to strong magnetic fields. Be- sides, exposure to strong magnetic fields $(\sim 8 \mathrm{~T})$ does not induce any harm in humans ${ }^{36)}$, and therefore, more research should explore the interactions between strong magnetic fields and skin, which may ultimately provide optimal treatments for the skin barrier.

\section{Acknowledgment}

The author is grateful to all members of the research and development department of Chifure Group, namely, Hiroka Maruyama and Makiko Kawana, for their kind assistance in the human study. The author is also grateful to Prof. Takanori Fukushima and Dr. Takashi Kajitani, of Tokyo Institute of Technology, for their valuable contributions in the magnetic research on liquid crystalline composites.

\section{References}

1) van der Gucht, J. Grand challenges in soft matter physics. Front. Phys. 6, 87 doi: 10.3389/fphy.2018.00087 (2018).

2) Meeker, S.P.; Poon, W.C.K.; Crain, J.; Terentjev, E.M. Colloid-liquid-crystal composites: An unusual soft solid. Phys. Rev. E. 61, 6083-6086 (2000).

3) Ishiwari, F.; Shoji, Y.; Fukushima, T. Supramolecular scaffolds enabling the controlled assembly of functional molecular units. Chem. Sci. 9, 2028-2041 (2018).

4) Hajjaj, F.; Kajitani, T.; Ohsumi, H.; Tanaka, Y.; Kato, K. et al. Rewriting the phase diagram of a diamagnetic liquid crystal by a magnetic field. Nat. Commun. 9, 4431 doi: 10.1038/s41467-018-06976-7 (2018).

5) Drzaic, P.S. Liquid Crystal Dispersions. World Scientific, Singapore (1995).

6) Sakamoto, K.; Lochhead, R.Y.; Maibach, H.I.; Yamashita, Y. Cosmetic Science and Technology: Theoretical Principles and Applications. Elsevier Inc., The Netherlands (2017).

7) Erb, R.M.; Martin, J.J;; Soheilian, R.; Pan, C. Actuating soft matter with magnetic torque. Adv. Funct. Mater. 26, 3859-3880 (2016).

8) Rikken, R.S.M.; Nolte, R.J.M.; Maan, J.C.; van Hest, J.C.M.; Wilson, D.A. et al. Manipulation of micro- and nanostructure motion with magnetic fields. Soft Matter, 10, 1295-1308 (2014).

9) Micali, N.; Engelkamp, H.; van Rhee, P.G.; Christianen, P.C.M.; Scolaro, L.M. et al. Selection of supramolecular chirality by application of rotational and magnetic forces. Nat. Chem. 4, 201-207 (2012).

10) Liu, M.; Ishida, Y.; Ebina, Y.; Sasaki, T.; Hikima, T. et al. An anisotropic hydrogel with electrostatic repulsion between cofacially aligned nanosheets. Nature, 517, 68-72 doi:10.1038/nature14060 (2015).

11) Rešetič, A.; Milavec, J.; Zupančič, B.; Domenici, V.; Za- 
lar, B. Polymer-dispersed liquid crystal elastomers. Nat. Commun. 7, 13140 doi: 10.1038/ncomms13140 (2016).

12) Honjo, S.; Yokota, M.; Doki, N.; Shimizu, K. Magnetic field influence on the crystal structure of 2,2':6,2"-terpyridine. Kagaku Kogaku Ronbunshu, 34, 383-387 (2008).

13) Potticary, J.; Terry, L.R.; Bell, C.; Papanikolopoulos, A.N.; Christianen, P.C.M. et al. An unforeseen polymorph of coronene by the application of magnetic fields during crystal growth. Nat. Commun. 7, 11555 doi: 10.1038/ ncomms11555 (2016).

14) Alam, M.A.; Motoyanagi, J.; Yamamoto, Y.; Fukushima, T.; Kim, J. et al. "Bicontinuous cubic" liquid crystalline materials from discotic molecules: A special effect of paraffinic side chains with ionic liquid pendants. $J$. Am. Chem. Soc. 131, 17722-17723 (2009).

15) Hayashi, H. Chemical reactions and magnetic fields. IEEE Translat. J. Magn. Jpn. 7, 586-590 (1992).

16) Chren, M.M.; Lasek, R.J.; Quinn, L.M.; Mostow, E.N.; Zyzanski, S.J. Skindex, a quality-of-life measure for patients with skin disease: Reliability, validity, and responsiveness. J. Invest. Dermatol. 107, 707-713 (1996).

17) Someya, T. Building bionic skin. IEEE Spectr. 50, 5056 doi: 10.1109/MSPEC.2013.6587191 (2013).

18) Ishiwari, F.; Hasebe, H.; Matsumura, S.; Hajjaj, F.; Hayashi, N.H. et al. Bioinspired design of a polymer gel sensor for the realization of extracellular $\mathrm{Ca}^{2+}$ imaging. Sci. Rep. 6, 24275 doi: 10.1038/srep24275 (2016).

19) Lee, S.E.; Lee, S.H. Skin barrier and calcium. Ann. Dermatol. 30, 265-275 doi: org/10.5021/ad.2018.30.3.265 (2018).

20) Yu, B.; Kang, S.Y.; Akthakul, A.; Ramadurai, N.; Pilkenton, M. et al. An elastic second skin. Nat. Mater. 15, 911-918 doi: 10.1038/NMAT4635 (2016).

21) Uchiyama, M.; Nagasawa, H.; Sasaoka, S.; Gabe, Y.; Toujou, T. et al. Skin on skin: Development of directelectrospinning fine-fiber technology. Proceedings of the $25^{\text {th }}$ IFSCC Conference, Milan, Italy (2019).

22) Bouwstra, J.A.; Honeywell-Nguyen, P.L.; Gooris, G.S.; Ponec, M. Structure of the skin barrier and its modulation by vesicular formulations. Prog. Lipid Res. 42, 1-36 (2003).

23) Bouwstra, J.A.; Ponec, M. The skin barrier in healthy and diseased state. Biochim. Biophys. Acta, Biomembr. 1758, 2080-2095 (2006).

24) Kang, M.; Tuteja, M.; Centrone, A.; Topgaard, D.; Leal,
C. Nanostructured lipid-based films for substrate-mediated applications in biotechnology. Adv. Funct. Mater. 28, 1704356 doi: 10.1002/adfm.201704356 (2018).

25) Gomez, M.P.; Leal, C. Lipid-based liquid crystalline films and solutions for the delivery of cargo to cells. Liq. Cryst. Rev. 7, 167-182 doi: org/10.1080/21680396.2 019.1666752 (2019).

26) Hajjaj, F.; Maruyama, H.; Kawana, M. Polymer dispersed liquid crystal film as a functional and aesthetic second skin barrier. J. Soc. Cosmet. Chem. Jpn. 55, 4 (in press).

27) Iwai, H.; Fukasawa, J.; Suzuki, T. A liquid crystal application in skin care cosmetics. Int. J. Cosmet. Sci. 20, 87-102 (1998).

28) Friberg, S.E. Micelles, microemulsions, liquid crystals, and the structure of stratum corneum lipids. J. Soc. Cosmet. Chem. 41, 155-171 (1990).

29) Hajjaj, F.; Maruyama, H.; Kawana, M.; Koyama, S.; Kamimura, K. Scalable production of wearable lipid-polymer composite films featuring skin-like properties via polymer-dispersed liquid crystal technology. Proceedings of the $31^{\text {st }}$ IFSCC Conference, Yokohama, Japan (2020).

30) Parsegian, V.A.; Rand, R.P.; Fuller, N.L.; Rau, D.C. Osmotic stress for the direct measurement of intermolecular forces. Methods Enzymol. 127, 400-416 (1986).

31) Bouwstra, J.A.; Gooris, G.S.; van der Spek, J.A.; Bras, W. Structural investigations of human stratum corneum by small-angle x-ray scattering. J. Invest. Dermatol. 97, 1005-1012 (1991).

32) Nakazawa, H.; Ohta, N.; Hatta, I. A possible regulation mechanism of water content in human stratum corneum via intercellular lipid matrix. Chem. Phys. Lipids, 165, 238-243 (2012).

33) Gorzelanny, C.; Goerge, T.; Schnaeker, E.M.; Thomas, K.; Luger, T.A. et al. Atomic force microscopy as an innovative tool for nanoanalysis of native stratum corneum. Exp. Dermatol. 15, 387-391 (2006).

34) Nowogrodski, C.; Simon, I.; Magdassi, S.; Shoseyov, O. Fabrication of second skin from keratin and melanin. Polymers, 12, 2568 doi: 10.3390/polym12112568 (2020).

35) Poinapen, D.; Toppozini, L.; Dies, H.; Brown, D.C.W.; Rheinstädter, M.C. Static magnetic fields enhance lipid order in native plant plasma membrane. Soft Matter, 9, 6804-6813 doi: 10.1039/c3sm50355k (2013).

36) Schenck, J.F. Safety of strong, static magnetic fields. J. Magn. Reson. Imaging, 12, 2-19 (2000). 